DAMTP-2001-52

hep-th/0106008

\title{
Brane Splitting via Quantum Tunneling
}

\author{
Selena $\mathrm{Ng}^{*}$ and Malcolm Perry ${ }^{\dagger}$ \\ Department of Applied Mathematics and Theoretical Physics, \\ Centre for Mathematical Sciences, \\ Wilberforce Road, Cambridge CB3 OWA, United Kingdom
}

\begin{abstract}
We study the two-centred $\mathrm{AdS}_{7} \times S^{4}$ solution of eleven-dimensional supergravity using the Euclidean path-integral approach, and find that it can be interpreted as an instanton, signalling the splitting of the throat of the M5 brane. The instanton is interpreted as indicating a coherent superposition of the quantum states corresponding to classically distinct solutions. This is a surprising result since it leads, through the AdS/CFT correspondence, to contradictory implications for the dual $(2,0)$ superconformal field theory on the M5 brane. We also argue that similar instantons should exist for other branes in ten- and eleven-dimensional supergravity. The counterterm subtraction technique for gravitational instantons, which arose from the AdS/CFT correspondence, is examined in terms of its applicability to our results. Connections are also made to the work of Maldacena et al on anti-de Sitter fragmentation.
\end{abstract}

*email: S.K.L.Ng@damtp.cam.ac.uk

†email: M.J.Perry@damtp.cam.ac.uk 


\section{Introduction}

Several years ago, Brill [1] considered an instanton connecting $\mathrm{AdS}_{2} \times S^{2}$ to a geometry containing two or more $\mathrm{AdS}_{2} \times S^{2}$ centres. Since $\mathrm{AdS}_{2} \times S^{2}$ is the near-horizon geometry of the extremal Reissner-Nordström black hole, Brill argued that the instanton describes the semi-classical splitting of the $\mathrm{AdS}_{2} \times S^{2}$ throat into two or more throats. It is well known that the extremal Reissner-Nordström black hole, and independently its throat $\operatorname{AdS}_{2} \times S^{2}$, can be considered as supersymmetric solutions of four-dimensional supergravity. In ten and eleven dimensions there exist analogous supersymmetric solutions of the supergravity action with anti-de Sitter near-horizon geometry, such as the D3 $\left(\mathrm{AdS}_{5} \times S^{5}\right), \mathrm{M} 2\left(\mathrm{AdS}_{4} \times S^{7}\right)$ and M5 $\left(\mathrm{AdS}_{7} \times S^{4}\right)$ branes. The natural question which then arises is whether the fragmentation process also occurs here in the context of string or M theory.

One might argue that fragmentation is forbidden on the grounds that each of these $\mathrm{AdS}_{p+2} \times S^{D-p-2}$ spacetimes is a supersymmetric solution of supergravity in $D$ dimensions, and thus stable. However, rather than indicating an instability, the existence of such an instanton indicates a quantum superposition of states. One expects that each classical solution of string or $\mathrm{M}$ theory is an approximation to the corresponding quantum state. Here we will consider the family of classical solutions with $n \mathrm{AdS}_{p+2} \times S^{D-p-2}$ centres! (for given $p$ and $D$ ), which can be thought of as analogous to the classical vacua of YangMills theory labelled by winding numbers $n$. In four-dimensional Yang-Mills theory, the existence of instantons connecting two vacua with two given winding numbers is interpreted as meaning that the true quantum vacuum, often referred to as the theta vacuum, is a linear superposition of the quantum states associated to each of the classical vacua. By analogy, we should interpret the instanton as indicating that the quantum state $\left|\psi_{n}\right\rangle$ associated to the $n$-centred $\mathrm{AdS}_{p+2} \times S^{D-p-2}$ geometry is not necessarily an exact eigenstate of the M theory "Hamiltonian". Instead we expect an exact eigenstate to be a coherent superposition of all the states $\left\{\left|\psi_{n}\right\rangle\right\}$.

However, a second and more serious objection to higher-dimensional AdS fragmentation

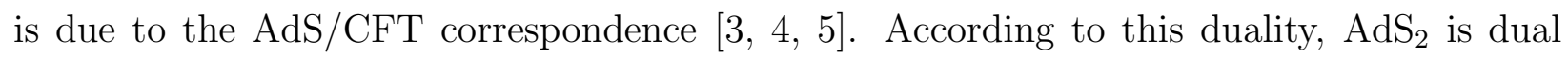
to quantum mechanics, and fields in quantum mechanics do not have well-defined vacuum expectation values, that is, they can fluctuate. Hence one would expect that the wavefunction describing the quantum state should be constructed as a superposition of each of the different classical vacua, which is in agreement with the existence of Brill's instanton. On the other hand, higher-dimensional AdS spaces are dual to quantum field theories. Scalar fields in field theories can, in contrast to quantum mechanics, be given fixed vacuum expectation values which are classical and constant. Since it costs an infinite action in field theory to change

\footnotetext{
${ }^{1}$ We will henceforth refer to these as $n$-centred $\mathrm{AdS}_{p+2} \times S^{D-p-2}$, although note that the geometry for $n \neq 1$ is not strictly a direct product, only approaching one-centred $\mathrm{AdS}_{p+2} \times S^{D-p-2}$ asymptotically.
} 
such vacuum expectation values dynamically, superposition of different exact vacua in the moduli space of the theory is forbidden. Translating to the AdS side, this would seem to imply that higher-dimensional analogues of the Brill instanton should not be allowed, if the AdS/CFT correspondence holds true.

Having established the above objections, it is somewhat surprising that we do identify in this paper, analogously to the four-dimensional case, an instanton which interpolates between the near-horizon $\mathrm{AdS}_{7} \times S^{4}$ geometry of the M5 brane and the "near-horizon" 2 two-centred $\mathrm{AdS}_{7} \times S^{4}$ geometry of the two-centred M5 brane. We postpone discussion on the resolution of this puzzle to the final section.

The standard procedure for identifying instantons, which we follow, is to use the Euclidean path integral approach to quantum gravity [6]. We will be interested in contributions to the zero-temperature vacuum amplitude, given by the path integral

$$
Z=\int D[\phi] e^{-I[\phi]}
$$

over all fields $\phi$ which are real on the Euclidean section and with boundary conditions appropriate to the zero-temperature vacuum, and where $I$ is the Euclidean action. In the semi-classical approximation, these contributions correspond to gravitational instantons, that is, nonsingular and geodesically complete solutions of the Euclidean equations of motion with finite action.

The Euclidean action is defined, for a metric $g_{a b}$ and $(p+1)$-form gauge potential $A_{p+1}$ on a $D$-dimensional manifold $M$ with boundary, as

$$
I=-\frac{1}{\kappa_{D}^{2}} \int_{M} d^{D} x \sqrt{g}\left(R-\frac{1}{2 \cdot(p+2) !} F_{p+2}^{2}\right)-\frac{2}{\kappa_{D}^{2}} \int_{\partial M} d^{D-1} x K \sqrt{h}-I_{0}[h] .
$$

$R$ is the Ricci scalar of the metric, $F_{p+2}=d A_{p+1}$ is the $(p+2)$-form field strength, $h_{a b}$ is the induced metric on the boundary, $K$ is the extrinsic curvature, and $\kappa_{D}^{2}=16 \pi G_{D}$ where $G_{D}$ is the $D$-dimensional gravitational constant. The surface integral is necessary in order to obtain an action which depends only on first derivatives of the metric and not normal derivatives to the boundary [7, 8], and ensures that the variational principle is well-defined. Finally, $I_{0}[h]$ is another surface term which depends only on the induced metric $h_{a b}$, and whose purpose will become clearer below.

Since the volume of anti-de Sitter space is infinite, the action (1.2) evaluated on an asymptotically $\mathrm{AdS}_{p+2} \times S^{D-p-2}$ space generally diverges. A common solution to this problem is to first regularise the action by imposing cut-offs, and then subtract the action $I_{0}$ of a background which acts as a "zero-point" and contains the same infrared divergences [9, 10]. This zero-point action must be written as a surface integral and corresponds exactly to the

\footnotetext{
${ }^{2}$ We mean this in the sense of [2] where the limit $L_{p} \rightarrow 0$ is taken, and $L_{p}$ is the Planck length $\left(L_{p}^{D-2}=\right.$ $\left.G_{D}\right)$.
} 
term $I_{0}[h]$ in (1.2) above. However this background subtraction technique is not in general well-defined. If for a particular geometry there exists a reference background with the same intrinsic metric on the boundary, the process works. Unfortunately, one cannot always find such a reference background. This is indeed the case for the multi-centred $\mathrm{AdS}_{p+2} \times S^{D-p-2}$ spacetimes.

The counterterm approach [11, 12, 13], which was motivated by the AdS/CFT correspondence, provides an alternative and well-defined procedure. Since the partition function of the string theory is conjectured to be equal to the generating functional of the dual conformal field theory, one can remove the divergences in the supergravity action by adding local counterterms on the boundary, giving a manifestly-finite, renormalised on-shell action. These local counterterms are proportional to surface integrals of the induced metric, and hence also take the form of the term $I_{0}[h]$ in (1.2]). As a side-remark, a consequence of this is that any interpolating, nonsingular, asymptotically AdS solution of the Euclidean field equations will have finite action, and can be interpreted as a gravitational instanton.

Much of the literature on using counterterm subtraction in Euclidean quantum gravity has focused on gauged supergravities obtained by reduction on a sphere of the tenor eleven-dimensional theory. However, the multi-centred $\mathrm{AdS}_{p+2} \times S^{D-p-2}$ solutions are warped geometries, due to the choice of direction connecting the two centres. This means that, as far as we are aware, the $D$-dimensional theory cannot be consistently reduced to a $(p+2)$-dimensional gauged supergravity, and thus the existing counterterms in [14] for asymptotically AdS spaces cannot be faithfully applied. An alternative would be to attempt to formulate the counterterms directly in the higher dimensions, and recent progress in this area has been made in [15, 16]. This will be discussed further in section 4 .

In the next section we will review the work of Brill on $\mathrm{AdS}_{2} \times S^{2}$, and in section 3 we present the $\mathrm{AdS}_{7} \times S^{4}$ instanton for the M5 brane using background subtraction. We will also compare our results with the claim in a paper by Maldacena et al [2] that brane creation by the field strength in supersymmetric AdS spaces occurs only for $\mathrm{AdS}_{2}$. In section 4 we examine the limitations of the background subtraction procedure, and discuss how these might be overcome by suitable counterterm regularisation. Section 5 contains preliminary results for the M2 and D3 branes, and a more conclusive result for the D1/D5 system, including a short discussion on correctly accounting for the contribution from self-dual field strengths to the instanton action. We also briefly speculate on nonconformal D branes and the NS5 brane. In the concluding section, we comment that our result for the M5 brane implies the superposition of distinct quantum vacua in the dual six-dimensional $\mathcal{N}=(2,0)$ superconformal field theory, in contradiction to the cluster decomposition principle, and speculate on its resolution. Note that all quantities, unless otherwise stated, will be presented in Euclidean signature. 


\section{The Brill Instanton}

For a one-form potential and metric in four dimensions, the supergravity action (1.2) reduces to

$$
I=-\frac{1}{\kappa_{4}^{2}} \int_{M} d^{4} x \sqrt{g}\left(R-\frac{1}{2 \cdot 2 !} F_{2}^{2}\right)-\frac{2}{\kappa_{4}^{2}} \int_{\partial M} d^{3} x K \sqrt{h}-I_{0}[h] .
$$

One family of solutions to the equations of motion of this action is the metric and two-form field strength

$$
\begin{aligned}
d s^{2} & =H^{-2} d t^{2}+H^{2} d s^{2}\left(\mathbb{E}^{3}\right), \\
F_{2} & =2 \star_{3} d H,
\end{aligned}
$$

where $H$ is a harmonic function on $\mathbb{E}^{3}$ and ' $\star_{3}$ ' is the Hodge dual also on $\mathbb{E}^{3}$. One recovers (one-centred) $\mathrm{AdS}_{2} \times S^{2}$ from the metric (2.2) by choosing

$$
H=\frac{b}{|\boldsymbol{x}|},
$$

where $b$ is a constant and $\boldsymbol{x}$ represents Cartesian coordinates on $\mathbb{E}^{3}$. The apparent singularity at $\boldsymbol{x}=0$ is a nonsingular horizon of the metric (2.2). In fact, the metric represents the near-horizon limit of the extremal Reissner-Nordström black hole with charge and mass $b$. Since the gravitational attraction between extremally charged black holes is balanced by the electric repulsion, another choice is the multi-centred harmonic function

$$
H=\sum_{k=1}^{n} \frac{b_{k}}{\left|\boldsymbol{x}-\boldsymbol{x}_{k}\right|}
$$

This corresponds to $n$ black holes each of charge (of equal sign) $b_{k}$ centred at $\boldsymbol{x}=\boldsymbol{x}_{k}$. Geometrically one has a spacetime which tends asymptotically to $\operatorname{AdS}_{2} \times S^{2}$ with radius $b_{k}$ whenever one of these centres is approached, that is, as $\boldsymbol{x} \rightarrow \boldsymbol{x}_{k}$, and to $\mathrm{AdS}_{2} \times S^{2}$ with radius $b_{1}+\ldots+b_{n}$ as the outer boundary $|\boldsymbol{x}| \rightarrow \infty$ is approached. This $n$-centred $\mathrm{AdS}_{2} \times S^{2}$ spacetime thus interpolates between the $n+1 \mathrm{AdS}_{2} \times S^{2}$ spaces. In the following we will restrict ourselves to the two-centred case $n=2$, however, it is straightforward to extend the analysis to general values of $n$.

Let us now, following Brill [1], compute the Euclidean action (2.1) for the two-centred $\mathrm{AdS}_{2} \times S^{2}$ geometry. As mentioned in the introduction, one expects the result to be infrareddivergent since AdS is noncompact. We will thus background subtract the reference action of one-centred $\mathrm{AdS}_{2} \times S^{2}$ in order to obtain a finite, meaningful result.

The metric equation of motion demands that the Ricci scalar vanishes in the action (2.1), reducing the bulk term to an integral of the two-form field strength. The source-free equation of motion $d * F_{2}=0$ for the field strength implies that one can write $\tilde{F}_{2} \equiv * F_{2}=d \tilde{A}$, for a one-form potential $\tilde{A}$. So the bulk term can be converted into

$$
\int_{M} d^{4} x \sqrt{g} \frac{1}{2 !} F_{2}^{2}=\int_{M} F_{2} \wedge * F_{2}=\int_{M} F_{2} \wedge d \tilde{A}=\int_{\partial M} F_{2} \wedge \tilde{A}+C,
$$


since $d F_{2}=0$ by the Bianchi identity. Note that although $\tilde{A}$ is only specified up to a gauge transformation, the surface integral above is obviously gauge-invariant. Here $C$ represents finite contributions to the action resulting from the non-smoothness of $\tilde{A}$. Since we are only interested in the finiteness of the action rather than its specific value, we will not explicitly evaluate these contributions here.

In spherical coordinates on $\mathbb{E}^{3}$ such that

$$
d s^{2}\left(\mathbb{E}^{3}\right)=d r^{2}+r^{2}\left(d \theta^{2}+\sin ^{2} \theta d \phi^{2}\right)
$$

the two-centred harmonic function takes the form

$$
H=\frac{b_{1}}{r_{1}}+\frac{b_{2}}{r_{2}}
$$

where

$$
r_{1}^{2}=r^{2}+a^{2}+2 a r \cos \theta, \quad r_{2}^{2}=r^{2}+a^{2}-2 a r \cos \theta .
$$

The two-form field strength, its dual and associated one-form potential are given by

$$
\begin{aligned}
F_{2} & =2 \partial_{r} H r^{2} \sin \theta d \theta \wedge d \phi+2 \partial_{\theta} H \sin \theta d \phi \wedge d r \\
\tilde{F}_{2} & =2 H^{-2} \partial_{r} H d t \wedge d r+2 H^{-2} \partial_{\theta} H d t \wedge d \theta \\
\tilde{A} & =2 H^{-1} d t
\end{aligned}
$$

Since the boundary of AdS lies at $r=\infty$ in these coordinates, we need to regularise the action by introducing an upper cutoff $R$ on the radial integration, and an upper cutoff $V_{1}$ on the temporal integration, then taking the limit $R \rightarrow \infty$ followed by $V_{1} \rightarrow \infty$ after performing background subtraction. The unit outward normal to the regularised boundary surface $r=R$ is $n=H^{-1} \partial_{r}$, giving the extrinsic curvature term

$$
K \sqrt{h} \equiv n \sqrt{h}=\left(r^{2} H^{-1} \partial_{r} H+2 r\right) \sin \theta .
$$

Thus the regularised action can be written in these coordinates as

$$
\begin{aligned}
\kappa_{4}^{2} I(R) & =\frac{1}{2} \int_{\partial M} F_{2} \wedge \tilde{A}-2 \int_{\partial M} d^{3} x K \sqrt{h}-\kappa_{4}^{2} I_{0}[h]+\frac{C}{2} \\
& =-4 \int_{r=R} d^{3} x\left(r+r^{2} H^{-1} \partial_{r} H\right) \sin \theta+\frac{C}{2} .
\end{aligned}
$$

Note that this vanishes identically for the one-centred geometry, making the background subtraction trivial. Substituting in the value of the two-centred harmonic function (2.8) and expanding in powers of $1 / R$ about $1 / R=0$, we obtain the result

$$
\kappa_{4} I(R)=-16 \pi V_{1}\left[R-\left(R+O\left(R^{-1}\right)\right)\right]+\frac{C}{2},
$$

which is finite in the limit $R \rightarrow \infty$ ! 
There are several points to note here. Firstly, although we are only interested in whether or not the action is finite and not in its exact value, we should point out that, as noted by Brill [1], the finite contributions result in the action giving the expected Bekenstein-Hawking entropy $S=A / 4$, where $A$ is the nonzero horizon area of the Reissner-Nordström black hole. The contribution to the path integral is then roughly $\exp (-2 I / \hbar)$ which is infinitely suppressed, as expected, in the classical limit $\hbar \rightarrow 0$.

Secondly, note the particular interpretation of the Brill instanton. In Lorentzian signature, the solution is static in time and inhomogeneous in space. In the Euclidean continuation, however, one can interpret the solution as being static in space and inhomogeneous in time (with a suitable redefinition of the coordinates as in [1]), since each spacetime direction now carries the same sign. The instanton thus describes being in an initial state (the onecentred universe) in one asymptotic region, and rolling to the final state (the two-centred universe) asymptotically. Both the initial and final states, being supersymmetric, are degenerate minima of the action of the theory - differing from the so-called "bounce" instanton solutions in which the solutions are saddlepoints and not genuine minima - and thus the quantum system is expected to be a superposition of these two states.

Thirdly, we have taken the magnetic solution (2.10) for the field strength $F_{2}$. We could also have chosen its electric dual $\tilde{F}_{2}$. This leads to a real instanton since in addition to the Wick rotation $t_{L} \rightarrow t_{E}=i t_{L}$, one analytically continues the electric charge $Q_{L} \rightarrow Q_{E}=i Q_{L}$, thus ensuring that fields which are real in Lorentzian space correspond to real fields on the Euclidean section [6].

Finally, note that the finite action is independent of the distribution of the charges, since the action vanishes independently of the choice of harmonic function - this agrees, by taking the limit $\left|b_{1}\right| \ll\left|b_{2}\right|$, with the result of Maldacena et al [2] where a one-centred $\mathrm{AdS}_{2} \times S^{2}$ universe was shown to fragment into a macroscopic universe and a microscopic brane. This can also be viewed as brane creation by the two-form field strength $F_{2}$ with charge proportional to $b_{1}$.

\section{The M5 Throat Instanton}

As mentioned in the introduction, one might expect that a similar instanton exists in the context of ten- or eleven-dimensional supergravity. It turns out that the simplest case to consider is the throat of the M5 brane in eleven-dimensional supergravity, since it is similarly nonsingular and couples magnetically to the three-form potential $A_{3}$ in the theory. The relevant bosonic action is

$$
\kappa_{11}^{2} I=-\int_{M} d^{11} x \sqrt{g}\left(R-\frac{1}{2 \cdot 4 !} F_{4}^{2}\right)+\int_{M} F_{4} \wedge F_{4} \wedge A_{3}-2 \int_{\partial M} d^{10} x K \sqrt{h}-\kappa_{11}^{2} I_{0}[h] .
$$


We are interested in the solution

$$
\begin{aligned}
d s^{2} & =H^{-1 / 3} d s^{2}\left(\mathbb{E}^{6}\right)+H^{2 / 3} d s^{2}\left(\mathbb{E}^{5}\right), \\
F_{4} & ={ }_{5} d H .
\end{aligned}
$$

$H$ is now a harmonic function on $\mathbb{E}^{5}$ and ' $\star_{5}$ ' is the Hodge dual on $\mathbb{E}^{5}$. The usual M5 brane solution is obtained by taking $H=1+b^{3} /|\boldsymbol{x}|^{3}$ where $\boldsymbol{x}$ are Cartesian coordinates on $\mathbb{E}^{5}, b$ is a constant, and the apparent singularity at $\boldsymbol{x}=0$ again corresponds to a horizon. If one discards the requirement of asymptotic flatness, then one can go directly to the "throat" or near-horizon geometry $\mathrm{AdS}_{7} \times S^{4}$ by setting

$$
H=\frac{b^{3}}{|\boldsymbol{x}|^{3}},
$$

and similarly for the $n$-centred geometry. The constant $b^{3}$ is now proportional to the charge or the number of M5 branes located at $\boldsymbol{x}=0$, and determines the radius $l$ of $\mathrm{AdS}_{7}$ by $l=2 b$.

We would like to evaluate the supergravity action for the two-centred $\operatorname{Ad} S_{7} \times S^{4}$ geometry analogously to the previous section. Choosing spherical coordinates on $\mathbb{E}^{5}$,

$$
d s^{2}\left(\mathbb{E}^{5}\right)=d r^{2}+r^{2}\left(d \theta^{2}+\sin ^{2} \theta d \Omega_{3}^{2}\right),
$$

where $d \Omega_{3}^{2}$ is the metric on the unit 3-sphere, we can write the two-centred harmonic function as

$$
H=\frac{b_{1}^{3}}{r_{1}^{3}}+\frac{b_{2}^{3}}{r_{2}^{3}},
$$

with $r_{1}, r_{2}$ defined as in (2.9). The metric equation of motion sets

$$
R=\frac{1}{6 \cdot 4 !} F_{4}^{2}
$$

As in the previous section, the bulk term can be converted into a surface integral. Noting that

$$
\begin{aligned}
K \sqrt{h} & =\left(\frac{1}{3} r^{4} H^{-1} \partial_{r} H+4 r^{3}\right) \sin ^{3} \theta \Theta_{3}, \\
F_{4} & =\partial_{r} H r^{4} \sin ^{3} \theta d \theta \wedge \operatorname{vol}\left(S^{3}\right)+\partial_{\theta} H r^{2} \sin ^{3} \theta d r \wedge \operatorname{vol}\left(S^{3}\right), \\
\tilde{F}_{7} & \equiv * F_{4}=d \tilde{A}_{6}=H^{-2} \partial_{r} H d r \wedge \operatorname{vol}\left(\mathbb{E}^{6}\right)+H^{-2} \partial_{\theta} H d \theta \wedge \operatorname{vol}\left(\mathbb{E}^{6}\right), \\
\tilde{A}_{6} & =-H^{-1} \operatorname{vol}\left(\mathbb{E}^{6}\right),
\end{aligned}
$$

where $\int \Theta_{3}=\Omega_{3}$, the volume of the unit three-sphere, and that the Chern-Simons term vanishes for the above solution, we can rewrite the regularised action as

$$
\begin{aligned}
\kappa_{11}^{2} I(R) & =\frac{1}{3} \int_{\partial M} F_{4} \wedge \tilde{A}_{6}-2 \int_{\partial M} d^{10} x K \sqrt{h}-\kappa_{11}^{2} I_{0}[h] \\
& =-\int_{r=R} d^{10} x\left(r^{4} H^{-1} \partial_{r} H+8 r^{3}\right) \Theta_{4}-\kappa_{11}^{2} I_{0}[h] .
\end{aligned}
$$


Introducing further upper cutoffs on the brane worldvolume directions by compactifying them on a six-torus with volume $V_{6}$, we obtain

$$
\kappa_{11}^{2} I(R)=-V_{6} \Omega_{4}\left(5 R^{3}+\frac{9}{5} \Delta_{3}^{2} a^{2} R\right)+O\left(R^{-1}\right)-\kappa_{11}^{2} I_{0}[h],
$$

where

$$
\Delta_{k}\left(b_{1}, b_{2}\right)=\frac{b_{1}^{k}-b_{2}^{k}}{b_{1}^{k}+b_{2}^{k}}
$$

describes the charge distribution of the configuration. This is clearly divergent in the limit $R \rightarrow \infty$, as we expect. In order to overcome this divergence, we will perform background subtraction, choosing as our "zero-point" the one-centred action

$$
\kappa_{11}^{2} I_{0}(R)=-5 V_{6} \Omega_{4} R^{3}
$$

The background-subtracted action is then

$$
\kappa_{11}^{2} I(R)=-\frac{9}{5} \Delta_{3}^{2} V_{6} \Omega_{4} a^{2} R+O\left(R^{-1}\right)
$$

which vanishes in the limit $R \rightarrow \infty$ followed by $V_{6} \rightarrow \infty$ only in the case that the charges are equally distributed, that is, $\Delta_{3}=0$, and otherwise diverges linearly in $R$ (and $V_{6}$ ).

How should we interpret this result? Analogously to Brill's instanton, with a suitable choice of coordinates, one can interpret the instanton as tunneling between a universe with two equally-charged centres and a universe with the same total charge, again at zero temperature. As we shall see below, the asymptotic boundary of two-centred $\mathrm{AdS}_{7} \times S^{4}$ in the case $b_{1}^{3}=b_{2}^{3}$ matches exactly to the boundary of one-centred $\operatorname{AdS}_{7} \times S^{4}$ with charge $b^{3}=b_{1}^{3}+b_{2}^{3}$, and thus the background subtraction is well-defined. This is not the case when $b_{1}^{3} \neq b_{2}^{3}$, where the background subtraction calculation must be taken with caveats. This means that although the above calculation suggests that no instanton exists which interpolates between a two-centred spacetime with $b_{1}^{3} \neq b_{2}^{3}$ and the one-centred geometry, we cannot conclusively assert this without applying a better-defined procedure such as the use of counterterms. Nevertheless, note that the result from background subtraction is consistent with the analysis of [2] in which the special case $\left|b_{1}\right| \ll\left|b_{2}\right|$ is considered. Here they find that fragmentation of a supersymmetric AdS universe into one macroscopic and one microscopic part, or the creation of a brane with charge given by $b_{1}$, is allowed only for $\mathrm{AdS}_{2}$, but not for higher-dimensional AdS spaces.

Note that, as opposed to the Brill instanton, in the case of the M5 brane the finite contribution vanishes since it is proportional to the horizon area, which vanishes for the M5 brane. Hence the zero action in this case correctly coincides with the zero entropy associated to the M5 brane. 


\section{The Need for Counterterms}

Let us examine in more detail how closely the boundary geometry of two-centred $\mathrm{AdS}_{p+2} \times$ $S^{D-p-2}$ matches that of the one-centred space, so that we can determine in which cases background subtraction can be faithfully applied. We will analyse this briefly for $\mathrm{AdS}_{2} \times S^{2}$ and $\mathrm{AdS}_{7} \times S^{4}$, then outline the counterterm technique and discuss its applicability to the M5 throat instanton action.

\subsection{Boundary Matching in Background Subtraction}

For $\mathrm{AdS}_{2} \times S^{2}$, the induced metric on the boundary is

$$
d s^{2}=H^{-2} d t^{2}+H^{2} R^{2} d \Omega_{2}^{2}
$$

in the limit $R \rightarrow \infty$, and where $H$ in the above equation is understood to be a function of the regulatory constant $R$ rather than the coordinate $r$. The $S^{2}$ radius for two-centred $\mathrm{AdS}_{2} \times S^{2}$

$$
H R=\left(\frac{b_{1}}{R_{1}}+\frac{b_{2}}{R_{2}}\right) R
$$

tends exactly to the one-centred $S^{2}$ radius $b$ at the boundary, provided that charge conservation holds, that is, $b_{1}+b_{2}=b$. Note that $R_{1}, R_{2}$ are defined as $r_{1}, r_{2}$ in (2.9) with $r$ replaced by $R$. However, the scale factor of the worldvolume coordinates

$$
H^{-2}=\left(b_{1}+b_{2}\right)^{-2}\left[R^{2}+2 \Delta_{1} a R \cos \theta+a^{2}\left(1-3\left(1-\Delta_{1}^{2}\right) \cos ^{2} \theta\right)\right]+O\left(R^{-1}\right)
$$

does not match asymptotically, where $\Delta_{1}$ is defined as in (3.8). The leading order term agrees with the one-centred value of $R^{2} / b^{2}$ (with charge conservation), but one is left with non-vanishing terms for any value of $\Delta_{1}$, that is, regardless of charge distribution! Despite this non-matching, however, the results in section 2 suggest that background subtraction still reproduces the correct result. We shall see in a few paragraphs that this is indeed the case.

Let us now turn to $\mathrm{AdS}_{7} \times S^{4}$. Here the boundary metric is

$$
d s^{2}=H^{-1 / 3} d s^{2}\left(\mathbb{E}^{6}\right)+H^{2 / 3} R^{2} d \Omega_{4}^{2}
$$

in the limit $R \rightarrow \infty$. The $S^{4}$ radius for two-centred space matches that for the one-centred

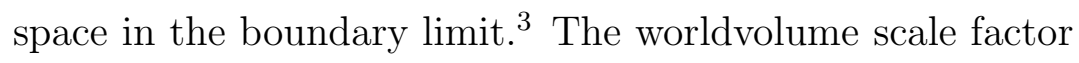

$$
H^{-1 / 3}=\left(b_{1}^{3}+b_{2}^{3}\right)^{-1 / 3}\left[R+\Delta_{3} a \cos \theta\right]+O\left(R^{-1}\right)
$$

\footnotetext{
${ }^{3}$ In fact, one can show that the $S^{D-p-2}$ radius for the two-centred $\operatorname{AdS}_{p+2} \times S^{D-p-2}$ always tends to the corresponding one-centred value in the boundary limit.
} 
again does not in general match asymptotically, except if $\Delta_{3}=0$, that is, when $b_{1}^{3}=b_{2}^{3}$ ! For unequal distributions, however, one is left with a finite term which renders the background subtraction procedure untrustworthy. So although the results of section 3 imply that no instanton exists for a two-centred spacetime with $b_{1}^{3} \neq b_{2}^{3}$, we cannot state this definitively without applying a better-defined procedure. Such a procedure is, as mentioned in the introduction, counterterm subtraction.

\subsection{Counterterms and the M5 Throat Instanton}

Counterterm subtraction has been employed successfully to calculate gravitational actions and thermodynamics of black holes such as the Taub-NUT-AdS, Taub-Bolt-AdS [13], and Kerr-AdS [17, for which the appropriate reference background was either unknown or ambiguous. In each of these cases, background subtraction provided a good approximation to the results obtained using counterterms, in some cases coinciding.

The idea behind this technique is as follows. The AdS/CFT correspondence equates the partition functions of the two theories. In the low energy limit, one can thus use the supergravity action in string theory to calculate the generating functional of the conformal field theory on the boundary. It turns out that the divergences which appear in the gravitational action are local integrals on the boundary [5], and thus can be removed by subtracting local surface counterterms. The form of these counterterms has been developed in [11, 12]. In particular, the counterterms to the purely gravitational action are (for $0 \leq p \leq 5)[13]$

$$
\begin{aligned}
I_{c t}=-\frac{2}{\kappa_{p+2}^{2}} \int_{\partial M} d^{p+1} x \sqrt{h}\left[\frac{p}{l}\right. & +\frac{l \mathcal{R}}{2(p-1)} \\
& \left.+\frac{l^{3}}{2(p-3)(p-1)^{2}}\left(\mathcal{R}_{a b} \mathcal{R}^{a b}-\frac{p+1}{4 p} \mathcal{R}^{2}\right)\right]+I_{\log }
\end{aligned}
$$

where $M$ is the asymptotically $\mathrm{AdS}_{p+2}$ space, $\mathcal{R}$ is the Ricci scalar of the induced metric $h$ on the boundary $\partial M$, and $I_{\log }$ is a logarithmically divergent term corresponding to the

conformal anomaly [1] which only contributes in dimensions for which $p$ is odd. Notice that no counterterms are necessary for $p=0$, which corresponds to the action for the Brill instanton. The action above should be read such that for $p=1$ only the first term is included, for $p=2,3$ only the first two terms are included, and for $p=4,5$ all three terms are included. In addition, for odd values of $p$ there is a contribution from the logarithmic term $I_{\log }$. Recall that $l$ is the radius of AdS.

For the $\mathrm{AdS}_{7} \times S^{4}$ instanton, the relevant counterterm action is

$$
I_{c t}\left(\mathrm{AdS}_{7}\right)=-\frac{2}{\kappa_{7}^{2}} \int_{\partial A d S_{7}} d^{6} x \sqrt{h}\left[\frac{5}{2 b}+\frac{b \mathcal{R}}{4}+\frac{b^{3}}{8}\left(\mathcal{R}_{a b} \mathcal{R}^{a b}-\frac{3}{10} \mathcal{R}^{2}\right)\right]+I_{\log }
$$

since $l=2 b$ for the $\mathrm{AdS}_{7} \times S^{4}$ metric (3.2). As a first check we compute the counterterms for the one-centred $\mathrm{AdS}_{7} \times S^{4}$ action. Since the boundary of $\mathrm{AdS}_{7}$ is Ricci-flat, we have that 
$I_{\log }$ vanishes [11] and

$$
I_{c t}\left(\mathrm{AdS}_{7}\right)=-\frac{2}{\kappa_{7}^{2}} \int_{r=R} d^{6} x \sqrt{h} \frac{5}{2 b}=-\frac{V_{6}}{b^{4} \kappa_{7}^{2}} 5 R^{3} .
$$

To uplift to eleven dimensions we simply use that $\kappa_{11}^{2}=\kappa_{7}^{2} b^{4} \Omega_{4}$, giving

$$
I_{c t}\left(\mathrm{AdS}_{7} \times S^{4}\right)=-\frac{V_{6} \Omega_{4}}{\kappa_{11}^{2}} 5 R^{3},
$$

which is exactly the value of the one-centred $\mathrm{AdS}_{7} \times S^{4}$ action obtained in (3.9), as we expect.

The counterterms presented in (4.6) arise from including a source for the stress-energy tensor in the dual conformal field theory, that is, they only cancel divergences corresponding to the purely gravitational action. However, the supergravity action (3.1) also contains matter coupled to gravity in the form of the field strength $F_{4}$. One might wonder if additional counterterms are necessary. To check this, one should first determine which seven-dimensional fields correspond to the eleven-dimensional $F_{4}$. This can be done since the Kaluza-Klein truncation of eleven-dimensional supergravity to maximally gauged supergravity in seven dimensions with gauge group $S O(5)$ has been shown to be consistent in [18]. One should then apply the counterterm criterion for the seven-dimensional scalar fields and other matter, which was derived in [19, 20] by turning on a source for each dual operator in the conformal field theory and following the same approach as for pure gravity. Without explicitly undertaking this procedure, however, the fact that the desired matching is obtained by adding the gravitational counterterm alone suggests that further counterterms should not be required.

We would like to compute counterterms for the two-centred action. However this is not straightforward. The reason is that the counterterms presented in [11, 12, [13] have been formulated for asymptotically AdS spacetimes. In the case that the solution of interest takes the form (asymptotically $\left.\mathrm{AdS}_{p+2}\right) \times S^{D-p-2}$, one can in general reduce the $D$-dimensional theory to $(p+2)$-dimensional gauged supergravity, and find the analogous (asymptotically $\operatorname{AdS}_{p+2}$ ) solution. The counterterms which arise from the dual conformal theory living on the $(p+1)$ dimensional boundary can then be applied. Unfortunately the two-centred (and in general $n$-centred) $\mathrm{AdS}_{p+2} \times S^{D-p-2}$ spacetime is, as mentioned in the introduction, a warped geometry. Rather than being a direct product of an asymptotically AdS space with a sphere, it takes the form of asymptotically $\left(\operatorname{AdS}_{p+2} \times S^{D-p-2}\right)$. Certain asymptotically $\left(\operatorname{AdS}_{p+2} \times S^{D-p-2}\right)$ geometries, such as some continuous distributions of $p$-branes, can be consistently reduced to a solution of a $(p+2)$-dimensional supergravity [21, 22]. Indeed, the counterterm renormalisation technique was recently applied to such a solution in [23]. However, it is not clear to us that a discrete distribution of $p$-branes, as the $n$-centred $\operatorname{AdS}_{p+2} \times S^{D-p-2}$ ge- 
ometries describe, finds an analogue in a reduced $(p+2)$-dimensional supergravity円, and thus it would appear that one cannot apply the counterterms, as originally formulated, to this class of solutions. An alternative which is available to us is to attempt to formulate the counterterms directly in the eleven-dimensional theory, as was carried out in [15] for the Polchinski-Strassler solutions in type IIB supergravity, and in [16] for $\mathrm{AdS}_{3} \times S^{3}$ and $\mathrm{AdS}_{5} \times S^{5}$. We will not consider this here but leave it as a further direction to pursue.

\section{$5 \quad$ Other Brane Throat Instantons?}

In the introduction to this paper we mentioned that we expect the result for the M5 brane to hold analogously for other supersymmetric branes in ten- and eleven-dimensional supergravity. Here we will present preliminary results for the M2 and D3 branes using background subtraction, and a more concrete result for the D1/D5 system with a discussion on counterterms and subtleties in dealing with self-dual field strengths. We finish by briefly commenting on other nonconformal D branes and the NS5 brane.

\subsection{The M2 and D3 Branes}

Like the M5 brane, the M2 and D3 branes are nonsingular and have anti-de Sitter nearhorizon geometries $-\mathrm{AdS}_{4} \times S^{7}$ and $\mathrm{AdS}_{5} \times S^{5}$ respectively. However, the results achieved using background subtraction have been less conclusive. The M2 throat instanton action contains a divergent term of order $R^{4}$, while the action of the D3 throat instanton contains a divergent term of order $R^{2}$.

Despite this, we would argue that these results cannot provide the complete picture, and that if we are able to apply a suitable counterterm technique as mentioned above, we should recover results analogous to that for the M5 brane. To motivate this, let us analyse whether the boundary geometries match.

The boundary metric of the two-centred $\mathrm{AdS}_{4} \times S^{7}$ geometry corresponding to the twocentred M2 brane is, in the limit $R \rightarrow \infty$,

$$
d s^{2}=H^{-2 / 3} d s^{2}\left(\mathbb{E}^{3}\right)+H^{1 / 3} R^{2} d \Omega_{7}^{2},
$$

where

$$
H=\frac{b_{1}^{6}}{R_{1}^{6}}+\frac{b_{2}^{6}}{R_{2}^{6}} .
$$

The $S^{7}$ radius can easily be shown to match to the one-centred value, but the scale factor for the worldvolume coordinates is

$$
H^{-2 / 3}=\left(b_{1}^{6}+b_{2}^{6}\right)^{-4}\left[R^{4}+4 \Delta_{6} a R^{3} \cos \theta+O\left(R^{2}\right)\right]
$$

\footnotetext{
${ }^{4}$ This is because one would need to turn on an infinite number of chiral fields [24 to accommodate the candidate solution in a reduced supergravity.
} 
where $\Delta_{6}$ is defined as in $(3.8)$.

For the two-centred $\mathrm{AdS}_{5} \times S^{5}$ geometry corresponding to the two-centred D3 brane, we have

$$
d s^{2}=H^{-1 / 2} d s^{2}\left(\mathbb{E}^{4}\right)+H^{1 / 2} R^{2} d \Omega_{5}^{2},
$$

where

$$
H=\frac{b_{1}^{4}}{R_{1}^{4}}+\frac{b_{2}^{4}}{R_{2}^{4}} .
$$

The worldvolume scale factor is then

$$
H^{-1 / 2}=\left(b_{1}^{4}+b_{2}^{4}\right)^{-1 / 2}\left[R^{2}+2 \Delta_{4} a R \cos \theta+O(1)\right],
$$

with $\Delta_{4}$ defined as in (3.8).

A pattern should now emerge: if we consider (4.4), (5.2) and (5.4) (4.2) is an exception), we see that the higher the dimension of the sphere, the higher the power of $R$ at which the boundary geometries do not match $-R^{0}$ for the four-sphere, $R$ for the five-sphere, and $R^{3}$ for the seven-sphere. This corresponds to one power of $R$ less than the highest order divergent term left in each of the on-shell instanton actions after background subtraction! We would therefore argue that one should not trust the results obtained from background subtraction except for the M5 brane case with two equal centres (and, of course, the Brill instanton), and that one should expect an appropriate counterterm procedure to show that analogous instantons exist for the M2 and D3 branes, at least in the cases with two equal centres.

\subsection{The D1/D5 System and Self-Dual Field Strengths}

The D1/D5 system of type IIB supergravity is yet another with anti-de Sitter near-horizon geometry, in this case, $\mathrm{AdS}_{3} \times S^{3} \times \mathbb{E}^{4}$. The action for this system is

$$
\kappa_{10}^{2} I=-\int_{M} d^{10} x \sqrt{g}\left(R-\frac{1}{2 \cdot 3 !} F_{3}^{2}\right)-2 \int_{\partial M} d^{9} x K \sqrt{h}-\kappa_{10}^{2} I_{0}[h] .
$$

We are interested in the solution!

$$
\begin{aligned}
d s^{2} & =H^{-1} d s^{2}\left(\mathbb{E}^{2}\right)+H d s^{2}\left(\mathbb{E}_{(1)}^{4}\right)+d s^{2}\left(\mathbb{E}_{(2)}^{4}\right), \\
F_{3} & =d A_{2}+\star_{6} d A_{2}, \\
A_{2} & =i H^{-1} \operatorname{vol}\left(\mathbb{E}^{2}\right) .
\end{aligned}
$$

\footnotetext{
${ }^{5}$ The following is meant for arbitrary values of $\Delta_{k}$; choosing equal charge distribution $\Delta_{k}=0$ results in reducing the power of $R$ by one, hence resulting in exact matching at the boundary for the $\operatorname{AdS}_{7} \times S^{4}$ geometry with two equal centres.

${ }^{6}$ Since the three-form field strength is self-dual and thus carries both "magnetic" and "electric" components, it will necessarily become complex in the continuation to Euclidean space. The issue of how to interpret this remains unresolved. This comment also applies to the self-dual five-form field strength in the D3 brane solution above.
} 
The subscripts on the two $\mathbb{E}^{4}$ spaces above are simply for clarity. Here $\mathbb{E}^{2}$ is the space tangent to both branes, $\mathbb{E}_{(1)}^{4}$ is transverse to both branes, $\mathbb{E}_{(2)}^{4}$ is transverse to the D1 branes but tangent to the D5 branes, and ' $\star_{6}$ ' refers to the Hodge dual on the space $\mathbb{E}^{2} \times \mathbb{E}_{(1)}^{4}$. We will choose spherical coordinates on $\mathbb{E}_{(1)}^{4}$

$$
d s^{2}\left(\mathbb{E}_{(1)}^{4}\right)=d r^{2}+r^{2} d \Omega_{3}^{2},
$$

and compactify $\mathbb{E}_{(2)}^{4}$ onto a four-torus $T^{4}$ of volume $V_{4}$. The choice of harmonic function

$$
H=\frac{b^{2}}{r^{2}}
$$

corresponds to the special non-dilatonic case in which the D1 and D5 brane charges are equal and proportional to $b^{2}$, giving the metric (5.6) the geometry $\mathrm{AdS}_{3} \times S^{3} \times T^{4}$ with $b$ the radius of both $\mathrm{AdS}_{3}$ and $S^{3}$. We have chosen to study this particular case since the resulting geometry for the two-centred choice

$$
H=\frac{b_{1}^{2}}{r_{1}^{2}}+\frac{b_{2}^{2}}{r_{2}^{2}}
$$

is a direct product of asymptotically $\left(\mathrm{AdS}_{3} \times S^{3}\right)$ with $T^{4}$. This means that we can essentially disregard the four-torus and work in six dimensions, with a geometry analogous to the previous cases we have studied above. As before, $b_{1}$ and $b_{2}$ represent the charges of the two centres, and $r_{1}, r_{2}$ are defined as in (2.9).

As we did in section 2 for the Brill instanton and section 0 for the M5 throat instanton, let us now calculate the two-centred $\mathrm{AdS}_{3} \times S^{3} \times T^{4}$ Euclidean action using background subtraction. The bulk action vanishes by virtue of the equations of motion (for $R$ ) and self-duality of $F_{3}$.. Since

$$
K \sqrt{h}=\left(\frac{1}{2} r^{3} H^{-1} \partial_{r} H+3 r^{2}\right) \Theta_{3}
$$

where $\int \Theta_{3}=\Omega_{3}$, the unit three-sphere volume, we obtain

$$
\begin{aligned}
\kappa_{10}^{2} I & =-2 \int_{r=R} d^{9} x K \sqrt{h}-\kappa_{10}^{2} I_{0}[h] \\
& =-V_{2} V_{4} \Omega_{3}\left(4 R^{2}+\Delta_{2}^{2} a^{2}\right)+O\left(R^{-1}\right)-\kappa_{10}^{2} I_{0}[h],
\end{aligned}
$$

where $\Delta_{2}$ is defined as in (3.8) and the coordinates on $\mathbb{E}^{2}$ have been compactified onto a two-torus $T^{2}$ of volume $V_{2}$, which will be taken to infinity after background subtraction. Choosing the one-centred action

$$
\kappa_{10}^{2} I_{0}(R)=-4 V_{2} V_{4} \Omega_{3} R^{2}
$$

\footnotetext{
${ }^{7}$ In the Euclidean continuation, the standard self-duality formula becomes $F_{3}=i * F_{3}$, hence leading to the problem outlined in the previous footnote.
} 
as the "zero-point", we obtain the background-subtracted action

$$
\kappa_{10}^{2} I(R)=-V_{2} V_{4} \Omega_{3} \Delta_{2}^{2} a^{2}+O\left(R^{-1}\right) .
$$

Similarly to the M5 brane throat, the action only vanishes in the limit $R \rightarrow \infty$ followed by $V_{2}, V_{4} \rightarrow \infty$ if $\Delta_{2}$ vanishes, that is, in the case of equal charges. So, using background subtraction, there exists a zero-temperature instanton describing the splitting of one D1/D5 throat into two equally charged D1/D5 throats. Note that the background subtraction result for splitting into two throats of unequal charge distribution $\left(\Delta_{2} \neq 0\right)$ again does not contradict the result of [2], nor the more detailed analysis of [25].

How trustworthy is background subtraction in this case? The worldvolume coordinate scale factor for the two-centred geometry is

$$
H^{-1}=\left(b_{1}^{2}+b_{2}^{2}\right)^{-1}\left[R^{2}+2 \Delta_{2} a R \cos \theta+\left(1-4 \cos ^{2} \theta\left(1-\Delta_{2}^{2}\right)\right) a^{2}\right]+O\left(R^{-1}\right),
$$

and does not match the one-centred value $R^{2} / b^{2}$ for any value of $\Delta_{2}$. However, we would argue, as for the Brill instanton (4.2), that the result obtained by background subtraction for the equal-centred case is correct. Perhaps it is the low dimension of the transverse sphere, and hence the low exponent of the highest divergent term in the action, which results in the correct result via background subtraction.

As expounded in the previous section, the only rigorous way of calculating the action is to use counterterm subtraction, rather than background subtraction. $\mathrm{AdS}_{3}$ corresponds to $p=1$ in the action (4.5), giving the only relevant counterterms

$$
I_{c t}\left(\mathrm{AdS}_{3}\right)=-\frac{2}{\kappa_{3}^{2}} \int_{\partial A d S_{3}} d^{2} x \frac{\sqrt{h}}{b}+I_{l o g} .
$$

For the one-centred $\mathrm{AdS}_{3} \times S^{3} \times T^{4}$ spacetime, $I_{\text {log }}$ again vanishes and, using that $\kappa_{10}^{2}=$ $\kappa_{3}^{2} V_{2} V_{4} b^{2} \Omega_{3}$, we have

$$
I_{c t}\left(\mathrm{AdS}_{3} \times S^{3} \times T^{4}\right)=-\frac{V_{2} V_{4} \Omega_{3}}{\kappa_{10}^{2}} 2 R^{2} .
$$

This is half the value of the one-centred $\mathrm{AdS}_{3} \times S^{3} \times T^{4}$ action (5.12) calculated above! How do we account for this discrepancy?

In general, varying a given action involves integrating by parts to obtain a bulk term and a boundary term. Forcing the bulk term to vanish determines the equations of motion for the system, and the vanishing of the boundary term determines the boundary conditions which the fields must satisfy for that particular action.

However, it can happen that there is a solution which obeys these equations of motion derived from the action but which does not obey the specified boundary conditions. In these cases, to compute the correct action one needs to add boundary terms to the original action such that the variation of this modified action still reproduces the same equations of motion, but now determines boundary conditions which coincide with the given solution. 
In our particular case we should consider the variation of the $F^{2}$ term in the bulk action:

$$
\delta \int_{M} F \wedge * F=2 \int_{M} d(\delta A) \wedge * F= \pm 2 \int_{M} \delta A \wedge d * F+2 \int_{\partial M} \delta A \wedge * F .
$$

The choice of sign in the bulk term above corresponds to the rank of $A$. If the boundary term does not vanish when evaluated on a particular solution, then this suggests that to obtain the correct value of the on-shell action, one should really add to the action the boundary term

$$
-2 \int_{\partial M} A \wedge * F
$$

Of course in the case of $\mathrm{AdS}_{3} \times S^{3}$ there are some subtleties due to the lack of a covariant action incorporating the self-dual $F_{3}$, however, it turns out that

$$
-\frac{1}{2}\left(-2 \int_{\partial M} A_{2} \wedge * F_{3}\right)=2 V_{2} V_{4} \Omega_{3} R^{2}
$$

is precisely the right term to add to $I_{0}$ (5.12) in order to equate it with $I_{c t}$ (5.15).

As for counterterms in the two-centred case, recent progress in [16] seems to indicate that the counterterm renormalised actions for both the two-centred $\operatorname{AdS}_{3} \times S^{3}$ and $\operatorname{AdS}_{5} \times S^{5}$ geometries do indeed vanish.

\subsection{Nonconformal D Branes and the NS5 Brane}

Although the near-horizon geometry of the supersymmetric $\mathrm{D} p$ brane $(p \neq 3)$ solutions of type II supergravity is only conformal to $\mathrm{AdS}_{p+2} \times S^{8-p}$ (or $\mathbb{E}^{(6,1)} \times S^{3}$ for $p=5$ ), one can still apply the same analysis above by working in what is called the dual frame [26]. This is defined as the frame in which the metric and the dual $(8-p)$-form field strength couple to the dilaton in the same way, and it is in this frame that all $\mathrm{D} p$ branes (except for $p=5$ ) have $\mathrm{AdS}_{p+2} \times S^{8-p}$ as their near-horizon geometry. It has also been argued [26] that the dual frame is holographic in the sense that taking the decoupling limit of the $\mathrm{D} p$ brane solution leads directly to a supergravity description, so it would be convenient to compute quantities in this frame in order to easily make statements about the corresponding conformal field theory.

One final brane for which it might be interesting to perform the same analysis is the supersymmetric NS5 brane in type IIA/B supergravity. Its near-horizon geometry is no longer anti-de Sitter, rather it is $S^{3} \times \mathbb{R} \times \mathbb{R}^{5,1}$, and the string theory in the NS5 brane background is conjectured 27] to be dual to the NS5 brane worldvolume theory. This worldvolume theory, often referred to as a "little string theory", turns out to be in an appropriate limit a sixdimensional superconformal field theory with $\mathcal{N}=(2,0)$ for type IIA, J and $\mathcal{N}=(1,1)$ for type

\footnotetext{
${ }^{8}$ Although it is not clear to us why this should be so, taking $F_{3}^{2}=\left|d A_{2}^{2}\right|+\left|\star_{6} d A_{2}^{2}\right|$ rather than zero also gives precisely the right contribution to agree with the counterterm result.

${ }^{9}$ This is the same $(2,0)$ theory dual to $\mathrm{M}$ theory on $\mathrm{AdS}_{7} \times S^{4}$, see [28] and references therein for further details. We thank T. Dasgupta for clarifying this point.
} 
IIB. One can as before construct supersymmetric multi-centred near-horizon geometries, and compute the appropriate Euclidean action evaluated on these solutions.

We expect that similar results will follow for the branes discussed in this subsection, but leave this for future work.

\section{Speculations}

In his original paper [1], Brill conjectured the existence of an instanton which describes the fragmentation of the complete extremal Reissner-Nordström wormhole and agrees with the $\mathrm{AdS}_{2} \times S^{2}$ instanton in the interior throat region. Unfortunately this has not yet been realised. Nevertheless, we would similarly conjecture that an instanton which describes the splitting of the M5 brane with two equal centres, and not just its two-centred $\mathrm{AdS}_{7} \times S^{4}$ throat, also exists.

If this is true, then drawing from the analogy we made in the introduction, an interpretation would be that the exact eigenstate of the full M theory "Hamiltonian", which we label naively as corresponding to the M5 brane, is in fact a coherent superposition of the quantum states $\left|\psi_{n}\right\rangle$ associated to each of the $n$-centred M5 branes which reside in the same charge sector of the supersymmetry multiplet, so that charge conservation holds. Intuitively, one can motivate this since the BPS property of the branes means that there are no forces involved in separating them, and thus the two-centred or in general $n$-centred geometries with $n$ separate stacks of branes are equally stable configurations with the same total energy. The question which remains from our analysis is whether the "M5 brane" eigenstate also superposes states which correspond to a non-uniform, or unequal, distribution of branes in the stacks, as in the case of the extremal Reissner-Nordström black hole.

What does all this imply for the dual conformal field theory? The AdS/CFT correspondence tells us that $\mathrm{M}$ theory on $\mathrm{AdS}_{7} \times S^{4}$ is dual to a six-dimensional $\mathcal{N}=(2,0)$ superconformal field theory [3], about which little is known. Since, by the results of section 5, we expect a similar interpretation to apply to the $\mathrm{AdS}_{5} \times S^{5}$ throat of the D3 brane, we will discuss, in the following paragraph, the correspondence in terms of $\operatorname{AdS}_{5} \times S^{5}$ and $\mathcal{N}=4$ super Yang-Mills, which is much better understood. Nevertheless, one should read the comments below as applying to any pair of anti-de Sitter space and conformal field theory related by the correspondence, in particular, to $\mathrm{AdS}_{7} \times S^{4}$ and the $(2,0)$ superconformal field theory.

Now string theory on $\mathrm{AdS}_{5} \times S^{5}$ with one centre is dual to $\mathcal{N}=4$ Yang-Mills at the superconformal point, where the vacuum expectation values of all the scalar fields vanish. Similarly, string theory on $\mathrm{AdS}_{5} \times S^{5}$ with more than one centre (for clarity we will discuss the case of two centres) is dual to the conformal field theory at a point in its moduli space where some of the scalar fields have acquired a nonzero vacuum expectation value, hence breaking the gauge group in a manner described in 24]. That the metric on two-centred 
$\mathrm{AdS}_{5} \times S^{5}$ interpolates between one-centred $\mathrm{AdS}_{5} \times S^{5}$ at $r \approx \infty$ and a two-centred geometry at $r \approx 0$, corresponds to the renormalisation group (RG) flow from $\mathcal{N}=4$ Yang-Mills at the superconformal fixed point in the ultraviolet to $\mathcal{N}=4$ Yang-Mills at some fixed point in the infrared.⿴囗⿰丨丨丁口⿹

However, this is not what we believe the instanton on the supergravity side implies for the conformal field theory. As touched on in the introduction, the fact that the transition amplitude between the one-centred and two-centred $\operatorname{AdS}_{5} \times S^{5}$ spaces is nonvanishing implies that the true vacuum of $\mathcal{N}=4$ Yang-Mills is actually a superposition of vacua in which the scalar fields have different vacuum expectation values. This is depicted in the figure below. On the other hand, one expects the vacua above to be exact vacua of the full quantum $\mathcal{N}=4$ Yang-Mills theory, and hence their superposition to be forbidden by the cluster decomposition principle. How can we understand this contradiction?

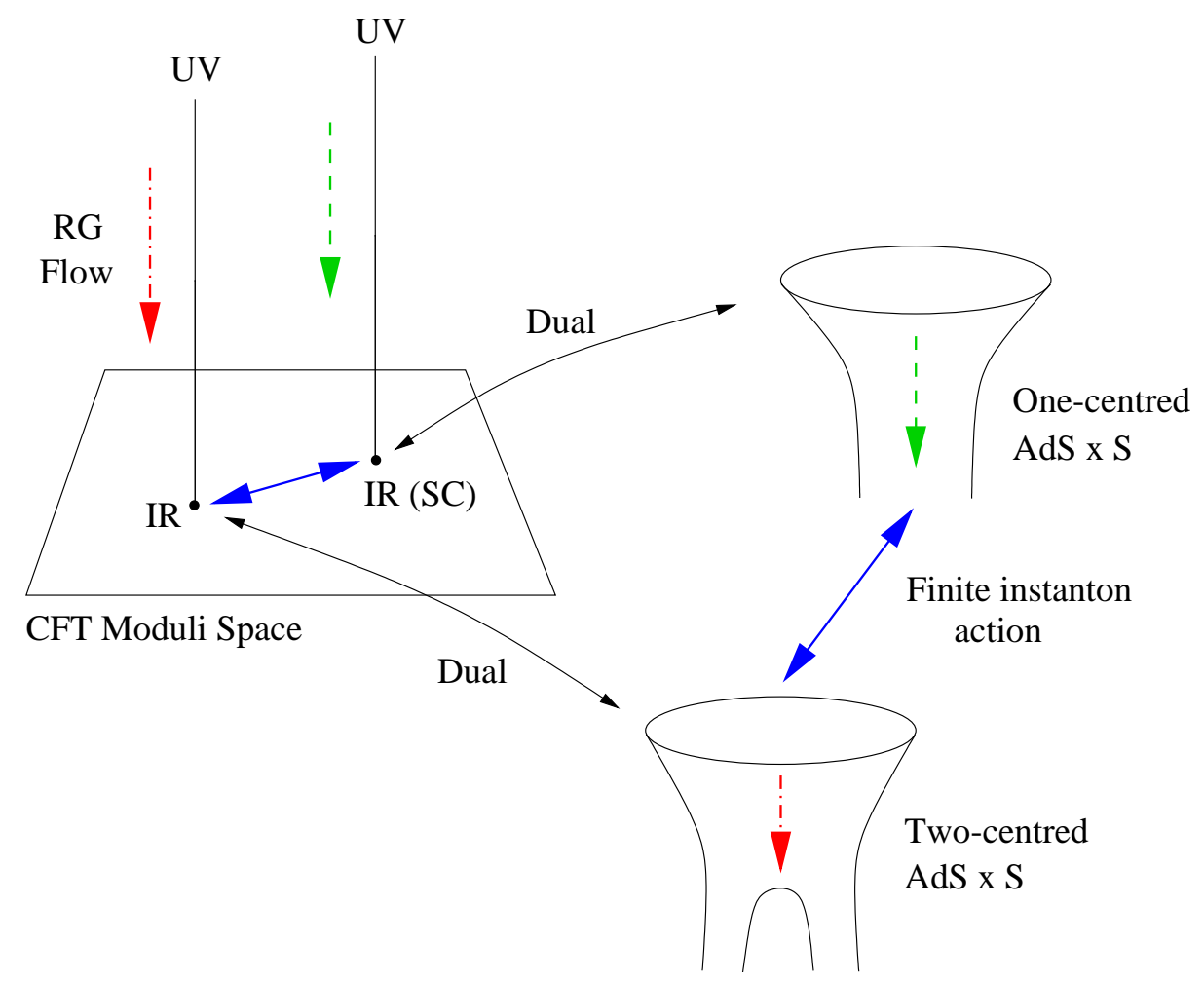

Figure 1: On the left hand side is the CFT picture, with the infrared fixed points shown, one of which is superconformal (SC). The dual AdS picture is shown on the right hand side. The dashed (green) and dashed/dotted (red) arrows represent RG flow from the ultraviolet (UV) to the infrared (IR) for the one-centred and two-centred AdS $\times S$ spaces respectively, while the plain (blue) arrow represents the nonzero transition amplitude between the one-centred and two-centred states in both pictures.

\footnotetext{
${ }^{10}$ For one-centred $\mathrm{AdS}_{5} \times S^{5}$ the flow is trivial.
} 
One should keep in mind that the result presented in this paper was calculated in semiclassical supergravity, and that while this serves as a low-energy approximation to M theory, it may be that $\mathrm{M}$ theory finds some way of resolving the contradiction by suppressing the tunneling, so that the effective contribution of such instantons to the amplitude vanishes. Since the full $\mathrm{M}$ theory is not yet known, it is possible that some as-yet-unknown symmetry not present in the low-energy supergravity description may impose a new superselection rule, preventing the tunneling process. It would be very interesting to investigate just how such a suppression might occur. Since the contradiction only arises in applying the AdS/CFT duality, one could also question if there is a subtlety in its application here to supergravity. However, we are inclined to believe that the correspondence holds true, and that the tunneling will be suppressed in the full $M$ theory. Yet another possibility is that the tunneling is instead suppressed even at the supergravity level by the presence of fermionic zero modes This issue is presently under investigation and we hope to report on it soon, for both the Brill instanton and the M5 throat instanton.

Finally, we would like to comment on the implications of our results for the issue of black hole entropy. Some years ago, it was claimed [29, 30, 31] using semiclassical topological arguments, that all extremal black holes, regardless of horizon area, should have zero entropy, and that the Bekenstein-Hawking area law applies only to nonextremal black holes. Since the Hawking temperature of extremal black holes vanishes, the zero entropy claim is supported by the third law of thermodynamics in its strongest form. While the extremal M5, M2 and D3 branes are in accordance with both the area law and the zero entropy claim due to their vanishing horizon areas, the extremal Reissner-Nordström black hole forces a contradiction.

On the other hand, string theory appears to favour the area law, and the thesis that extremal black holes should be obtained as limits of their nonextremal counterparts. The counting of the microscopic quantum degrees of freedom corresponding to the black hole entropy was pioneered by Strominger and Vafa in [32]. When applied to the Reissner-Nordström black hole, the string analysis results in the expected nonzero Bekenstein-Hawking entropy [33. There was an attempt to resolve this apparent contradiction between the macroscopic and microscopic counting of the entropy in [34].

As mentioned above, the extremal M5, M2 and D3 branes each have vanishing horizon area and hence vanishing entropy. This agrees with the fact that each of the above branes is BPS (supersymmetric), and thus there should be no quantum corrections to the semiclassical entropy as given by the Bekenstein-Hawking area law. Furthermore, an object with vanishing entropy should truly be fundamental, in the sense that it has no constituent degrees of freedom. The extremal M5, M2 and D3 branes can indeed be considered to fall into this category.

\footnotetext{
${ }^{11}$ We thank M. Bianchi for pointing this out, and for discussions regarding this.
} 


\section{Acknowledgments}

S.N. would like to thank M. Bianchi, G. W. Gibbons, H. S. Reall, K. Skenderis, M. M. TaylorRobinson and P. K. Townsend for interesting discussions. In particular, S.N. is grateful to D. Mateos for many valuable comments, many more enlightening conversations, and for a careful reading of the manuscript. Finally, we would like to thank a referee for useful and insightful comments. The work of S.N. was supported by Churchill College Cambridge and an ORS Award.

\section{References}

[1] D. R. Brill, Splitting of an Extremal Reissner-Nordström Throat via Quantum Tunneling, Phys. Rev. D 46 (1992) 1560, hep-th/9202037.

[2] J. Maldacena, J. Michelson, A. Strominger, Anti-de Sitter Fragmentation, J. High Energy Phys. 02 (1999) 011, hep-th/9812073.

[3] J. M. Maldacena, The Large N Limit of Superconformal Field Theories and Supergravity, Adv. Theor. Math. Phys. 2 (1998) 231, hep-th/9711200.

[4] S. Gubser, I. Klebanov, A. Polyakov, Gauge Theory Correlators from Noncritical String Theory, Phys. Lett.B 428 (1998) 105, hep-th/9802109.

[5] E. Witten, Anti De Sitter Space And Holography, Adv. Theor. Math. Phys. 2 (1998) 253, hep-th/9802150.

[6] S. W. Hawking in General Relativity, an Einstein Centenary Survey, eds. S. W. Hawking and W. Israel, Cambridge University Press, 1979.

[7] J. W. York Jr., Role of Conformal Three-Geometry in the Dynamics of Gravitation, Phys. Rev. Lett. 28 (1972) 1082.

[8] G. W. Gibbons, S. W. Hawking, Action Integrals and Partition Functions in Quantum Gravity, Phys. Rev. D 15 (1977) 2752.

[9] S. W. Hawking, D. N. Page, Thermodynamics of Black Holes in Anti-de Sitter Space, Commun. Math. Phys. 87 (1983) 577.

[10] E. Witten, Anti-de Sitter Space, Thermal Phase Transition, And Confinement In Gauge Theories, Adv. Theor. Math. Phys. 2 (1998) 505, hep-th/9803131.

[11] M. Henningson, K. Skenderis, The Holographic Weyl Anomaly, J. High Energy Phys. 07 (1998) 023, hep-th/9806087. 
[12] V. Balasubramanian, P. Kraus, A Stress Tensor for Anti-De Sitter Gravity, Commun. Math. Phys. 208 (1999) 413, hep-th/9902121.

[13] R. Emparan, C. V. Johnson, R. C. Myers, Surface Terms as Counterterms in the AdS/CFT Correspondence, Phys. Rev. D 60 (1999) 104001, hep-th/9903238.

[14] K. Skenderis, Asymptotically Anti-de Sitter Spacetimes and their Stress Energy Tensor, Int. J. Mod. Phys. A 16 (2001) 740, hep-th/0010138.

[15] M. Taylor-Robinson, Anomalies, Counterterms and the $\mathcal{N}=0$ Polchinski-Strassler Solutions, hep-th/0103162.

[16] M. Taylor-Robinson, Higher-Dimensional Formulation of Counterterms, hep-th/0110142.

[17] A. M. Awad, C. V. Johnson, Holographic Stress Tensors for Kerr-AdS Black Holes, Phys. Rev. D 61 (2000) 084025, hep-th/9910040; Higher Dimensional Kerr-AdS Black Holes and the AdS/CFT Correspondence, Phys. Rev. D 62 (2000) 125010, hep-th/0008211.

[18] H. Nastase, D. Vaman, P. van Nieuwenhuizen, Consistent Nonlinear KK Reduction of 11d Supergravity on $A d S_{7} \times S^{4}$ and Self-Duality in Odd Dimensions, Phys. Lett.B 469 (1999) 96, hep-th/9905075.

[19] S. de Haro, K. Skenderis, S. N. Solodukhin, Holographic Reconstruction of Spacetime and Renormalization in the AdS/CFT Correspondence, Commun. Math. Phys. 217 (2001) 595, hep-th/0002230.

[20] M. Taylor-Robinson, More on Counterterms in the Gravitational Action and Anomalies, hep-th/0002125.

[21] D. Z. Freedman, S. S. Gubser, K. Pilch, N. P. Warner, Continuous Distributions of D3branes and Gauged Supergravity, J. High Energy Phys. 07 (2000) 038, hep-th/9906194.

[22] M. Cvetič, S. S. Gubser, H. Lü, C. N. Pope, Symmetric Potentials of Gauged Supergravities in Diverse Dimensions and Coulomb Branch of Gauge Theories, Phys. Rev. D 62 (2000) 086003, hep-th/9909121.

[23] M. Bianchi, D. Z. Freedman, K. Skenderis, How to go with an RG Flow, hep-th/0105276.

[24] I. R. Klebanov, E. Witten, AdS/CFT Correspondence and Symmetry Breaking, Nucl. Phys. B 556 (1999) 89, hep-th/9905104.

[25] N. Seiberg, E. Witten, The D1/D5 System and Singular CFT, J. High Energy Phys. 04 (1999) 017, hep-th/9903224. 
[26] H. J. Boonstra, K. Skenderis, P. K. Townsend, The Domain-Wall/QFT Correspondence, J. High Energy Phys. 01 (1999) 003, hep-th/9807137.

[27] O. Aharony, M. Berkooz, D. Kutasov, N. Seiberg, Linear Dilatons, NS5-Branes and Holography, J. High Energy Phys. 10 (1998) 004, hep-th/9808149.

[28] O. Aharony, S. S. Gubser, J. Maldacena, H. Ooguri, Y. Oz, Large N Field Theories, String Theory and Gravity, Phys. Rep. 323 (2000) 183, hep-th/9905111.

[29] G. W. Gibbons, R. E. Kallosh, Topology, Entropy and Witten Index of Dilaton Black Holes, Phys. Rev. D 51 (1995) 2839, hep-th/9407118.

[30] S. W. Hawking, G. T. Horowitz, S. F. Ross, Entropy, Area and Black Hole Pairs, Phys. Rev. D 51 (1995) 4302, hep-th/9409013.

[31] C. Teitelboim, Action and Entropy of Extreme and Non-Extreme Black Holes, Phys. Rev. D 51 (1995) 4315, hep-th/9410103.

[32] A. Strominger, C. Vafa, Microscopic Origin of the Bekenstein-Hawking Entropy, Phys. Lett.B 379 (1996) 99, hep-th/9601029.

[33] J. M. Maldacena, A. Strominger, Statistical Entropy of Four-Dimensional Extremal Black Holes, Phys. Rev. Lett. 77 (1996) 428, hep-th/9603060.

[34] S. Das, A. Dasgupta, P. Ramadevi, Can Extremal Black Holes have Nonzero Entropy?, Mod. Phys. Lett. A 12 (1997) 3067, hep-th/9608162. 